\title{
An automatic simulation tool for thermal analysis of gravity dams by BFM
}

\author{
Jianming Zhang \\ State Key Laboratory of Advanced Design and Manufacturing \\ for Vehicle Body, Hunan University, China
}

\begin{abstract}
The dam's construction schedule has significant influences in long-term concrete temperature, whose variations may produce local thermal stress and thus result in damage and cracking. This paper describes a methodology for predicting the thermal evolution in large concrete placements with special emphasis on gravity dams during their construction by the Boundary Face Method (BFM). A simulation tool that completely integrated into a CAD environment (here UG$\mathrm{NX}$ is adopted) has been developed. Benchmark examples are presented and compared with other solutions by the FEM.
\end{abstract}

Keywords: gravity dam, boundary face method, transient thermal analysis, hierarchical matrix, $A C A$.

\section{Introduction}

It is widely recognized by engineers that the high temperature generated in the concrete due to the hydration heat of the cement paste is one of the main factors that result in fractures and damage for concrete hydraulic structures during their construction. The Finite Element Method (FEM) is the main approach for analyzing the thermal state of the dams. Various aspects of thermal stresses in concrete dams have been investigated and reported by the FEM. However, the results reported by different researchers are usually different or even contradict to each other. The reason for this may owe to that the FEM has the following intrinsic drawbacks:

1) Many kinds of abstract element, e.g. beam element, shell element, bar element, spring element, etc., are used in the FEM (usually up to hundreds of elements included in a mature commercial FEM software package). These 
elements are all based on some mathematic assumptions. Selection of proper element type and reasonable explanation to the computational results require sound theoretical background, good knowledge of all elements performance and rich experience in numerical analysis.

2) The analysis automation of the FEM is presently at very low level. Too many important parameters related to computation method have to be selected by the analysts. As the FEM model (approximate grid model) is completely different from the CAD model (continuous parametric model), not only in geometry and topology, but also in representation data structure. This makes the interaction between $\mathrm{CAE}$ and $\mathrm{CAD}$ extremely difficult. A truly seamless interaction is determinatively impossible.

3) The task of proper meshing for the FEM analysis is challenging and keeps the most critical part of the analysis. The accuracy of the FEM is highly dependent on the quality of the mesh. To make a dam meshable, the geometry of the dam structure is often simplified. In the simplification, small sized features at the connecting area between different parts of the structure are simply omitted. These features, however, are most possibly the places where local stress concentrations take place and cracks originate from. Moreover, further assumptions are required to connect different kinds of elements in the assembled matrix. This leads to much worse accuracy for the stresses or even wrong results, and thus makes the entire analysis unbelievable and useless.

4) The FEM is based on the equivalent weak form of the governing equation and the boundary conditions of the BVP. The trial functions in the FEM formulation must be at least $\mathrm{C} 1$ continuous. This leads to a number of contradictions, e.g. the contradiction between conforming and non-conforming elements, accurate integration and locking problems, reduced integration and hourglass modes, accuracy and stability in penal function method, etc. Moreover, errors of the FEM come from not only the approximation of the trial function but also from the "weak form" theory. Meanwhile, for a stress analysis by FEM, as stresses are calculated from the derivatives of displacements, the accuracy for stresses is lower by one order than displacements. In many cases, however, engineers are more concerned by the stress values.

To overcome the above problems, we have put forward the concept of Complete Solid Stress Analysis (CSSA) [1] and proposed the Boundary Face Method (BFM) [2, 3] based on the Boundary Integral Equation (BIE) [4] to carry out the CSSA. In most cases, the BFM requires only boundary mesh, which can be obtained by discretizing each piecewise continuous panel of the body's surface without restriction of element connectivity, hence, considerably simplifies the discretization task. Moreover, it uses the parametric representation of domain surfaces, only. Such representation is used in any CAD software and can be accessed in commercial packages via Open Architecture features (usually the in-process COM servers/objects can be exploited). This may considerably simplify the data pre-processing and lead to substantial resources savings.

Our contributions are as follows:

1) Integrating the BFM into UG-NX, making the conductions of the analyses entirely within the CAD environment. 
2) Proposing two kinds of new elements, i.e. tube element and element with negative parts. These elements have been successfully used to deal with cooling water pipes in dam.

3) Proposing a domain number sequence optimization method for solving multi-domain problems, which can deal with arbitrary inter-domain connections and get best efficiency by optimizing the band of the assembling system matrix.

4) Proposing a geometric mapping cross approximation (GMCA) method [5], which is equivalent to ACA but without iteration. The GMCA makes the lowrank representation of the BIE more convenient and efficient.

5) Implementing a quasi-initial condition method for transient thermal problems, and proposing a time step scaling method to solve the instability problem occurs in case of short time steps. (This is the case for dam simulation, as the heat conductivity of concrete is small but its hydration speed is relatively high).

\section{Implementation of the BFM for dam simulation}

\subsection{Integration of the BFM into UG-NX}

A primary version of CSSA software (Potent 1.0) has been developed. This software is completely integrated into the environment of the well known CAD package UG-NX (see Fig. 1). So far, the Potent 1.0 is able to solve problems in theories of static elasticity [6], steady state [7] and transient heat transfer [8] and acoustics [9]. Details can be found at the web site: www.5aCAE.com.

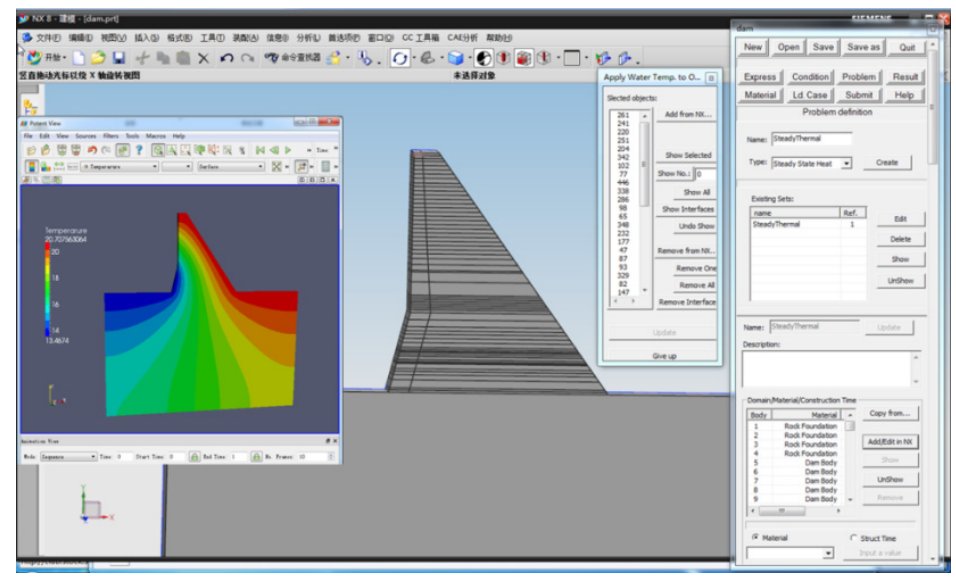

Figure 1: $\quad$ UI of the Potent1.0.

The Potent 1.0 is not only more accurate and efficient than most well known commercial CAE packages (e.g. NASTRAN, ANSYS, etc.), but also exhibits the following extraordinary merits: 
1) Analysis can be easily performed by clicking some buttons in the UI of the NX-UG. It is not necessary for the users to master the knowledge about computational mechanics but just knowledge about material mechanics. With correctly imposed boundary conditions, accurate results can be obtained.

2) Imposition of constraints and loads is also accomplished in the UI of the NX-UG. They are intuitively imposed on the CAD model rather than on the grid model. The mesh for the structure is automatically generated and invisible to the user. Therefore, the user does not need to care about meshing.

3) Accurate prediction of temperature at arbitrary points can be made on a complex structure, e.g. in the vicinity of the cooling water pipes. Case studies investigating how the construction schedule affects the temperature development can also be easily performed.

\subsection{Special elements for cooling water pipes}

To control the temperature development during a dam construction, a practical way is to embed cooling water pipes into some layers of the dam. The diameters of the water pipes are usually 3-4 centimetres, while dimensions of the dam are of hundreds of meters. Direct discretization of these pipes using traditional methods will result in prohibitively large amount of elements. This is the reason why in most FEM analysis, the specific shape and dimensions of the water pipes are simply omitted but instead, an equivalent heat sink is assumed to take into account the cooling effect of them. Obviously, the equivalent method will deteriorate the accuracy of the analysis, especially at points near the pipes. To consider the exact geometry of the pipes while keeping the computational scale under control so that the analysis can be conducted on a desktop computer, we proposed two kinds of special elements: the tube element and the triangle element with negative part [7,8]. Fig. 2 shows an example of meshing on a square face with five holes by the triangle elements with negative parts and traditional triangle elements, respectively. A validation example of a transient thermal problem solved by BFM and FEM is presented in Fig. 3. The results are in good agreement. However, the BFM computation used only 1656 nodes, while to get the same level of accuracy by the FEM, 129594 nodes are required. For details please refer $[7,8]$.

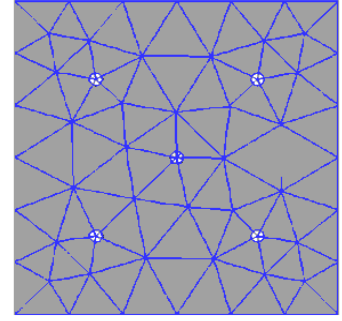

(a)

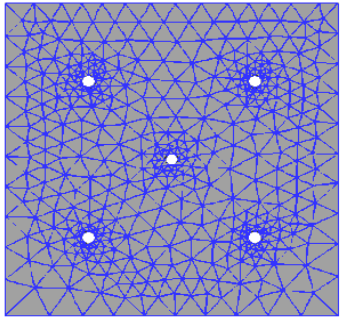

(b)

Figure 2: A face with five small holes. (a) Triangular mesh with new elements. (b) Traditional triangular mesh. 

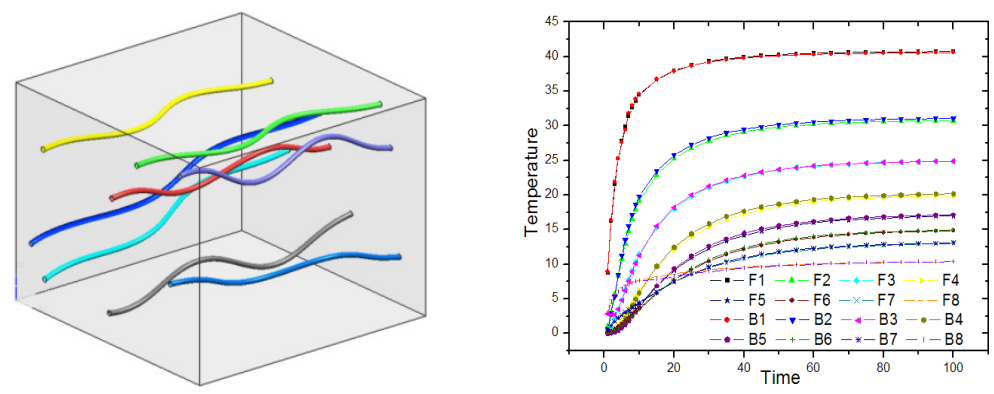

Figure 3: Comparison with finite element method.

\subsection{Domain number sequence optimization method for multi-domain problems}

In the dam construction simulation, the dam are divided into hundreds of layers, and the numbers of layers involved in each time steps of the analysis increases in the process of the dam elevation. Therefore, a multi-domain solver is a must for the simulation. Fortunately, a multi-domain formulation has a very beneficial advantage that it leads to an overall assembled coefficient matrix in the final system equations with a sparse blocked structure. The sparsity pattern is directly related to the number order of the domains. However, the domain number obtained from the UG model is usually in random order. In this work, a domain number optimization method is proposed. Fig. 4 shows an example of arbitrarily connected domains of a dam. The structure is discretized with 6868 quadratic triangle and quad elements and 21733 nodes. The optimized domain number sequence is $12,13,6,11,10,9,5,4,1,3,8,2$, 7, which is shown in Fig. 5. The sparsity patterns of the overall matrices before and after optimization are shown in Fig. 6. The time used for LU-decomposition of the overall matrix is $128 \mathrm{~s}$ and $54 \mathrm{~s}$, respectively. A big improvement of efficiency has been achieved. And it can be expected that with increasing the total number of nodes, the improvement will be more remarkable. A description in detail of the method in a journal paper form is in preparing.

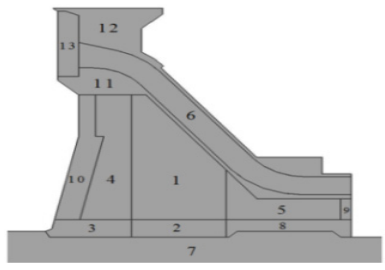

Figure 4: Domain numbers of a concrete dam.

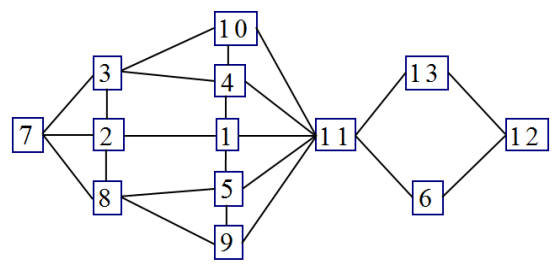

Figure 5: Domain number sequence optimisation. 

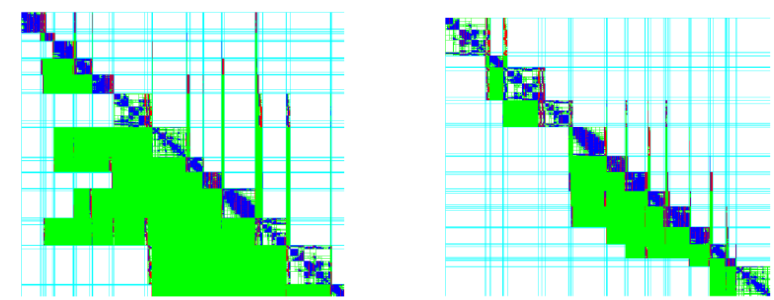

Figure 6: The assembled overall matrices before and after optimization.

\subsection{Geometric mapping cross approximation method for low-rank representation}

Discretization of a gravity dam may result in a large number of elements, up to millions usually, making the computational scale extremely large. Among the so far available accelerating methods that can dramatically reduce the memory and computational time, the H-matrix combined with the ACA [10] is a purely algebraic algorithm, and can be easily integrated into existing BEM codes. Nevertheless, even with the reduced complexity of $\mathrm{O}(N \log N)$, the memory requirement for the H-matrix is much higher than that for the Fast Multipole Method (FMM) $[9,11]$. In this work, we have implemented the basic algebraic algorithms of the H-matrix in an out-of-core manner. Another difficulty is associated with the iterative feature of the ACA, in which the involved matrix entries are calculated during the iteration. In the conventional BEM, however, the boundary integration is carried out in a way that integration quantities are firstly computed for an element, then loop for all source nodes. Therefore, a noniterative version of ACA that could determine the skeleton points of the low rank matrix before computing the boundary integrals would be desirable and beneficial. To this end, we proposed a geometric mapping cross approximation (GMCA) [5]. Our method is based on a geometric mapping operation, which just needs some simple geometric transformations. By the GMCA the procedure of low rank approximation and the evaluation of boundary integrals are separated. In contrast, the original ACA does them in one routine and, therefore, would be inconvenient and inefficient in the cases where the matrix entries need to be numerically evaluated. This is why so far most fast BEM implementations are based on constant elements. Three validation examples of the GMCA are presented in Fig. 7. The results are compared with the ACA in Table 1. For details please refer [5].

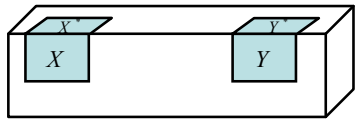

(a)

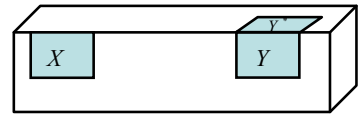

(b)

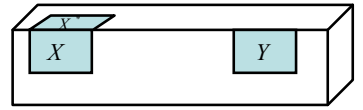

(c)

Figure 7: Geometries for the examples with single layer potential gradients. 
Table 1: $\quad$ Numerical results obtained by GMCA and ACA.

\begin{tabular}{lllllll}
\hline & \multirow{2}{*}{$\mathrm{m}$} & $\mathrm{n}$ & \multicolumn{2}{c}{ New algorithm } & \multicolumn{2}{c}{ ACA } \\
\cline { 5 - 6 } & & $\mathrm{k}$ & Accuracy & $\mathrm{k}$ & Accuracy \\
\hline (a) & 800 & 800 & 4 & $1.452 \times 10^{-3}$ & 5 & $3.885 \times 10^{-2}$ \\
(b) & 400 & 800 & 2 & $3.137 \times 10^{-4}$ & 4 & $4.606 \times 10^{-6}$ \\
(c) & 800 & 400 & 2 & $2.448 \times 10^{-5}$ & 4 & $7.943 \times 10^{-6}$ \\
\hline
\end{tabular}

\subsection{Time step scaling in quasi-initial condition method of transient thermal analysis}

A quasi-initial condition method has been implemented to solve the time domain boundary integral equation in the dam transient thermal analysis. Because of the high hydration speed of concrete, the time steps used in the analysis have to be relatively small. On the other hand, as the heat conductivity of concrete is very small, large time steps are necessary to maintain the computation numerically stable. To overcome this difficulty, a time step scaling method is proposed. The time step is firstly amplified and the temperature and the fluxes are computed at the end point of the amplified step, namely a virtual time point. The boundary conditions at the virtual time point are determined through a linear interpolation using the conditions at the real time point and the quasi-initial time. The heat sources is assumed to be constant and taken as the same value as in the real time step. Finally, the temperature and the fluxes at the virtual time point are linearly interpolated back to the real time step. A theoretical verification of the time scaling method can be found in [12]. Fig. 8 shows an example of transient heat conduction in a cube. In the case that the time step equals to $0.1 \mathrm{~s}$, the result is wrong. However, when the time step is amplified by 2, 4 and 8 times, the results converge to the exact solution.
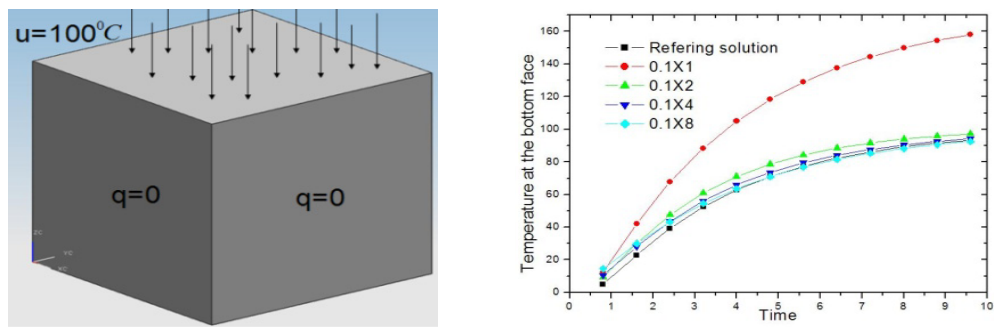

Figure 8: Transient heat conduction in a cube.

\section{Results for thermal simulation of dams}

Several numerical examples for thermal analysis of dams are presented and compared with other solutions by commercial FEM tools, e.g. ANSYS or ABACUS. The examples involve steady state heat conduction, transient heat 
conduction and heat simulation in varying domains (the construction simulation). Results have demonstrated the advantages of the BFM with respect to both accuracy and efficiency.

\subsection{Steady state heat conduction in a dam}

In old ages, the thermal state in a dam approaches stable. At this stage, the temperature distribution in it can be predicted by a steady state heat conduction analysis. Fig. 9 shows a sliced part of a concrete dam with the width being $20 \mathrm{~m}$. The air temperature is taken as $20.4^{\circ} \mathrm{C}$. The temperature of water is given by $T_{w}=20.7-0.0591572 \times h$, where $h$ is the depth into the water. All other faces are prescribed as adiabatic boundary condition. The material properties for the dam and base are given in Table 2 . In the table $\mathrm{c}, \rho$ and $\kappa$ are the heat capacity, the mass density and the heat conductivity, respectively.

Table 2: $\quad$ Material properties for the dam and rock foundation.

\begin{tabular}{llll}
\hline & $\mathrm{c}$ & $\rho$ & $\kappa$ \\
& $k J /\left(k g{ }^{\circ} \mathrm{C}\right)$ & $\mathrm{kg} / \mathrm{m}^{3}$ & $\mathrm{~kJ} /\left(\mathrm{m} \cdot \mathrm{h}^{\circ} \mathrm{C}\right)$ \\
\hline Dam & 2400 & 0.9627 & 9.27 \\
Base & 2450 & 0.9627 & 8.776 \\
\hline
\end{tabular}

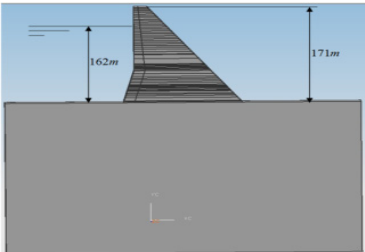

Figure 9: The dam model defined in UG-NX.

The temperature distribution is shown in Fig. 10. And Fig. 11 shows the results by ABACUS. The BFM computation used totally 29139 nodes while the FEM computation used 272300 nodes.

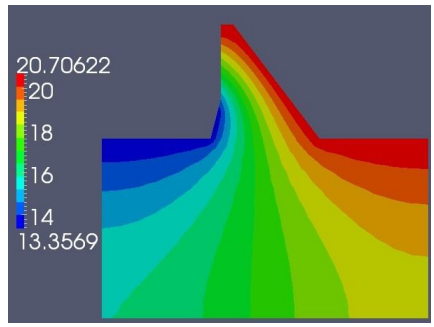

Figure 10: The thermal state by Figure 11: The thermal state by BFM.

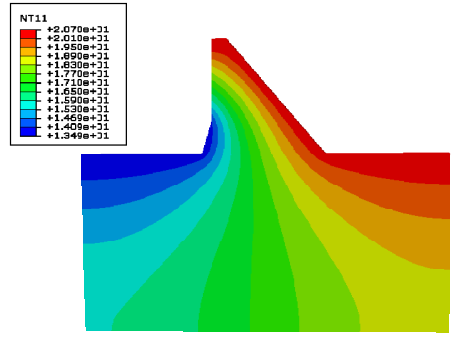

FEM. 


\subsection{Transient heat conduction in a dam}

This example considers a natural cooling process of a dam shown in Fig. 12. The dam model consists of two parts: the dam body and the base part (the rock foundation). The heat capacity, the mass density and the heat conductivity for both the dam and the foundation are $2539 \mathrm{~kg} / \mathrm{m}^{3}, 0.888 \mathrm{~kJ} /\left(\mathrm{kg}{ }^{\circ} \mathrm{C}\right)$ and $9.75 \mathrm{~kJ} /\left(\mathrm{m} \cdot h{ }^{\circ} \mathrm{C}\right)$, respectively. The initial temperature for the dam is $20^{\circ} \mathrm{C}$ and for the base is $0^{\circ} \mathrm{C}$. The environmental temperature is also $0^{\circ} \mathrm{C}$. The model is discretized with 3896 boundary elements and 2418 nodes. The considered time span is 8 years, which is divided into 384 analysis time steps. For comparison, this problem has also been solved using ANSYS11.0 with 1585 quadrilateral elements in two dimensions (Fig. 13). Temperature distributions at years 3 are compared in Figs 14-15. Again, the results are in very good agreement with each other.

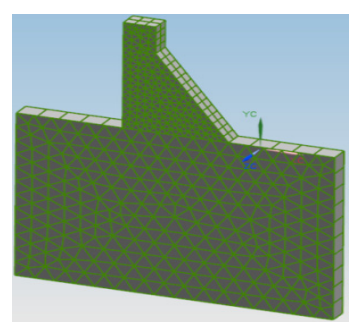

Figure 12: The BFM mesh.

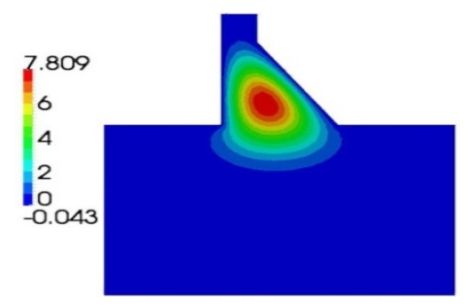

Figure 14: Thermal state by BFM.

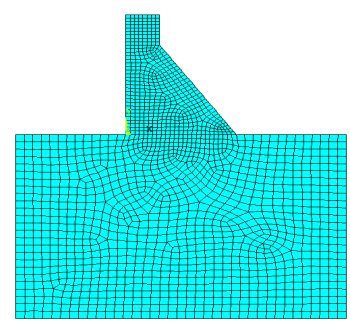

Figure 13: The FEM mesh.

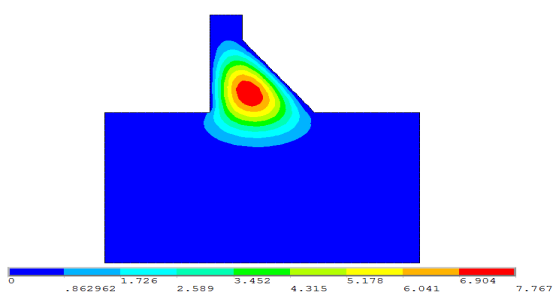

Figure 15: Thermal state by FEM.

\subsection{Transient thermal problems involving varying domains}

To simulate the temperature development during a gravity dam's construction process, a transient problem involving varying domains must be solved. This is because a gravity dam is constructed layer by layer. Preparation of FEM input data for such kind of problems could be very complicated. However, the simulation can be automatically carried out by our CAD/CAE unified solution scheme. In the scheme, the geometry model of the multi-layer dam is built in UG-NX, and the layers involved in every analysis step are defined according to the construction schedule. The interfaces between the layers are identified automatically in each analysis step and either the boundary condition or interface 
condition are also determined and imposed on the surfaces of the layers. An example of a simplified dam model containing 16 layers is considered here to validate our algorithms and solution scheme. To compare with the FEM, the problem has also been solved using ANSYS11.0. In the FEM computation, the model is further simplified into a bi-dimensional problem.

The heat diffusivity and the heat conductivity for both the dam and the base are $0.004 \mathrm{~m}^{2} / h$ and $10 \mathrm{~kJ} /\left(\mathrm{m} \cdot \mathrm{h}^{\circ} \mathrm{C}\right)$, respectively. The initial temperature for the whole model is $0^{\circ} \mathrm{C}$. The adiabatic temperature rise of the concrete layers is given by

$$
\theta(\tau)=\frac{25.0 \tau}{4.5+\tau}\left({ }^{\circ} \mathrm{C}\right)
$$

And the heat source generation rate in the rock foundation is 0 . The environmental temperature is also $0^{\circ} \mathrm{C}$. Fixed temperatures taken as the environmental temperature are prescribed on the bottom and right sides of the rock foundation. Adiabatic boundary conditions are imposed on all left side faces of the model. Surfaces exposed to atmosphere satisfy the Robin boundary condition:

$$
\lambda \frac{\partial T}{\partial n}=-\beta\left(T-T_{a}\right),
$$

where $T_{a}$ is the environmental temperature. The convection surface coefficient $\beta$ takes the value of $60.0 \mathrm{~kJ} /\left(\mathrm{m} \cdot \mathrm{h}^{\mathrm{o}} \mathrm{C}\right)$.

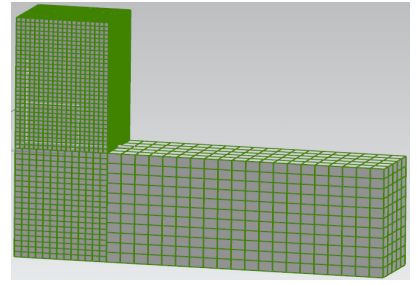

Figure 16: The BFM mesh for 16 layers.

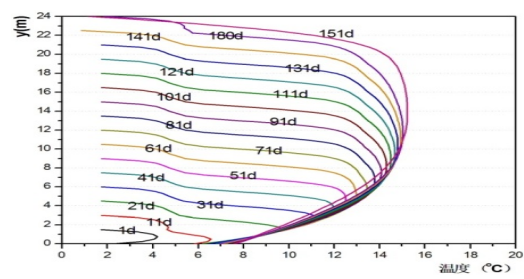

Figure 18: Temperature by BFM.

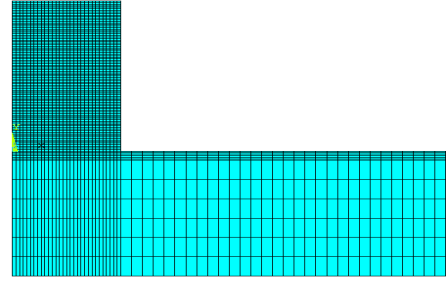

Figure 17: The FEM mesh for 16 layers.

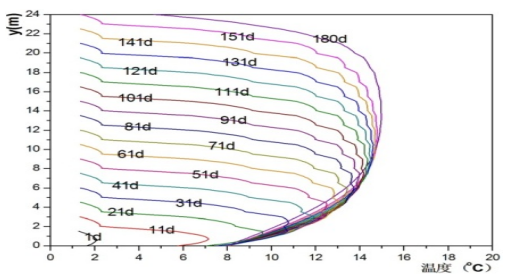

Figure 19: Temperature by FEM. 
The discretized models are show in Figs. 16 and 17. Totally 2379 boundary quadrilateral elements have been used for the BFM computation, and 450 planar quadrilateral elements for the FEM computation. Figs 18 and 19 represent the temperature variation during the time along the central vertical line on the left side of the model.

\subsection{A real dam simulation}

The gravity dam shown in Fig. 9 is considered in the present analysis. The dam is divided into 62 layers according to its construction schedule. The characteristics, such as materials, boundary and initial conditions, the hydration heat of the cement are taken as the example described in Section 3.3. The simulation consists of 60 sequential analyses, which are further subdivided into 1260 time steps, and span totally 76500 hours. For the simulation, 5827 boundary quadrilateral elements with 24527 nodes and 5360 hexahedron volume elements with 36327 internal nodes have been used. The entire computation is finished within 2107 seconds (with domain number sequence optimization). Figs. 20-21 show the temperature distribution in the dam body at the considered time. For further details and animation of the temperature development, see the web site: www.5aCAE.com.

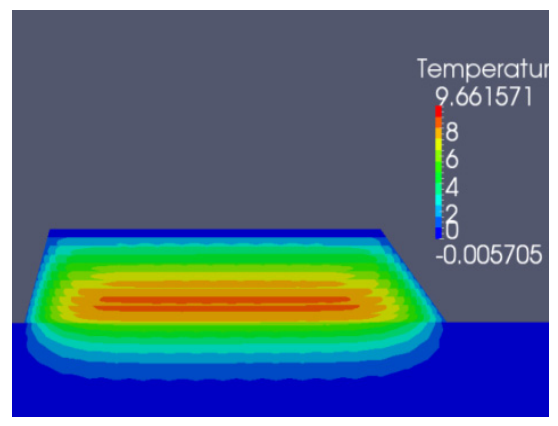

Figure 20: Thermal state at hour 100 .

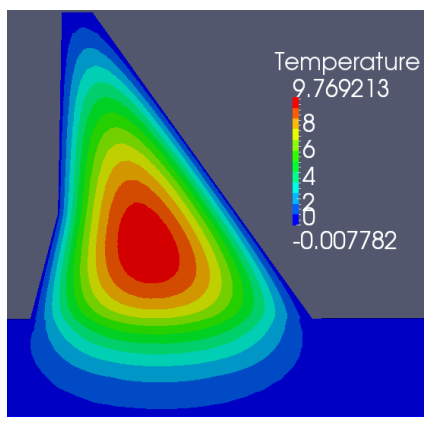

Figure 21: Thermal state at hour 900 .

\section{Conclusion}

In this work, the Boundary Face Method is applied in the computation of the temperature in gravity dams with heat generation due to hydration of concrete. The BFM has been seamlessly integrated into a CAD package (here the NX-UG has been chosen), making the thermal analysis can be automatically conducted and completely within the CAD environment. All the characteristics of analysis, such as the material properties, boundary and initial conditions, heat sources etc., are applied to the geometry entities naturally with the CAD tools, rather than to elements or nodes. 
Bench mark examples with comparison with some mature FEM packages (e.g. ABACUS and ANSYS) are presented. It has been demonstrated that our method is able to get high accuracy equivalent to the FEM but with lower cost in terms of both human labour and computational resources.

\section{Acknowledgement}

This work was supported by National Science Foundation of China under grant number 11172098 .

\section{References}

[1] 5aCAE, www.5aCAE.com.

[2] Zhang J.M., Qin X.Y., et al., A boundary face method for potential problems in three dimensions, International Journal for Numerical Methods in Engineering, 80, 320-337, 2008.

[3] Qin X.Y., Zhang J.M., Li G.Y., Sheng X.M., Song Q., A finite element implementation of the boundary face method for potential problems in three dimensions, Engineering Analysis with Boundary Element 34, 934-943, 2010.

[4] Brebbia, C.A., Telles, J.C.F. and Wrobel, L.C., Boundary Element Techniques, Springer-Verlag: Berlin and New York, 1984.

[5] Zhang J.M, Zheng X.S., A Geometric Mapping Cross Approximation Method. Computer Modeling in Engineering and Sciences. Submitted.

[6] Huang C., Zhang J.M, Qin X.Y., Lu C.J., Sheng X.M., Li G.Y. Stress analysis of solids with open-ended tubular holes by BFM. Engineering Analysis with Boundary Elements 36, 1908-1916, 2012.

[7] Qin X.Y., Zhang J.M., Liu L.P., Steady-state heat conduction analysis of solids with small open-ended tubular holes by BFM, International Journal of Heat and Mass Transfer, 55, 6846-6853, 2012.

[8] Zhou F.L., Xie G.Z., Zhang J.M., et al., Transient heat conduction analysis of solid with small open-ended tubular cavities by boundary face method, Engineering Analysis with Boundary Elements, 37, 542-550, 2013.

[9] Wang X.H., Zhang J.M., Zhou F.L., Zheng X.S. An adaptive fast multipole boundary face method with higher order elements for acoustic problems in three-dimension. Engineering Analysis with Boundary Elements, 37: 114 $-152,2013$.

[10] Bebendorf, M., Rjasanov, S., Adaptive Low-Rank Approximation of Collocation Matrices. Computing, 70(1), 1-24, 2003.

[11] Zhang J.M., Tanaka Masa., Adaptive spatial decomposition in fast multipole method, J. Comput. Phys., 226, 17-28, 2007.

[12] Zhou F.L., Li X.H., Zhang J.M., Huang C., Lu C.J, A time step amplification method in boundary face method for transient heat conduction, International Journal of Heat and Mass Transfer. Submitted. 